\title{
Computer-Assisted Target Control Infusion System for Elderly Patients during Endoscopic Retrograde Cholangiopancreateography
}

\author{
Kai Li ${ }^{1}$, Bin Zhang ${ }^{2, *}$, Zhihua Zhu ${ }^{1}$, Jingpeng $\mathrm{Jin}^{2}$ and Yang Liu ${ }^{2}$ \\ ${ }^{1}$ Anesthesiology Department \\ ${ }^{2}$ Endoscopic Center, China-Japan Union Hospital of Jilin University, 126th Xian-Tai Avenue, Changchun City, Jilin Province, \\ 130021, P.R.China \\ *corresponding author
}

\begin{abstract}
Objectives: The aim of this study was to investigate the safety and efficacy of propofol sedation with a computer-assisted target-controlled infusionsystem in elderly patients during endoscopic retrograde cholangiopancreateography (ERCP). Methods: Included were 150 consecutive EPCP procedures elderly patients under propofol sedation with a TCI system. Patients were divided into 3 groups: group $A$, age 50-70 years $(n=50)$; group $B$, age 71-80 years $(n=50)$; group $C$, age $>80$ years $(n=50)$. We compared the propofol dose and adverse events (hypotension and hypoxemia) during ERCP. Results: Older patients required a lower target concentration of propofol with higher age period: Median target concentration are respectively $2.6 \mu \mathrm{g} / \mathrm{mL}, 2.1 \mu \mathrm{g} / \mathrm{mL}$ and $1.6 \mu \mathrm{g} / \mathrm{mL}$ in the three groups. Hypotension tended to occur in the younger group, and hypoxemia occurred at a significantly higher rate in the older groups, although the number of cases was small. Low preoperative systolic blood pressure $(<80 \mathrm{~mm}$ $\mathrm{Hg}$ ) was associated with hypotension and abnormal pulmonary function was associated with hypoxemia in groups B and C. Conclusions: Elderly patients required lower doses of propofol with computer-assisted TCI system than younger patients. Attention to hypoxemia is necessary in elderly patients, particularly patients with abnormal pulmonary function.
\end{abstract}

Keywords-computer-assisted pump; target control infusion; endoscopic retrograde cholangiopancreateography

\section{INTRODUCTION}

Endoscopic retrograde cholangiopancreatography (ERCP) is commonly used in the management of many pancreatobiliary disorders. ERCP is the ideal method for extraction of common bile duct stones. it reduced the need for the more invasive surgical procedures associated with high morbidity particularly in

old age patients. ERCP with stent placement can be effective for the palliation of obstructive jaundice in pancreatic cancer which is commonly happened in elder patients [1]. In comparison with upper gastrointestinal endoscopic procedures, ERCP is a longer and more complex procedure, with a substantially higher complication rate [2]. Sedation for endoscopic procedures has gained much interest in recent years. Sedation obviously ensures comfort for the patients and endoscopists during the procedure, but sometimes sedation in ERCP may be responsible for some postoperative adverse events especially for elderly patients [3].The most commonly complications in ERCP with sedation including cardiopulmonary events such as hypoxemia, hypoventilation, airway obstruction, apnea, arrythmia, hypotension, and vasovagal episodes [4]. However, sedation for ERCP is popular especially when complex and painful interventions are planned in prone position.

It can be hypothesized that elderly patients require lower doses of sedation to achieve similar pharmacological effects compared with younger patients. A target-controlled infulion(TCI) system, which is the computer-assisted pump enables automatic control of the dose of sedative drugs by a infusion algorithm of pharmacokinetics for calculating the effect-site concentration[5] However, the pharmacokinetic model in the TCI pump may not be optimal when considering the age and concomitant disease in individual patients[6].

However, there is limited information on the outcomes of sedation used in elderly patients. This study aimed to evaluate the safety and efficacy of propofol sedation with appropriate amounts of propofol with the use of the TCI system for elderly patients during ERCP.

\section{METHODS}

\section{A. Patients and Study Design}

A total of 150 consecutive ERCP procedures for early patients were performed at the endoscope center of China-Japan union hospital of Jilin university by using propofol sedation with a comoputer-assisted TCI system between October 2013 and September 2014 were included in this study. Patients were divided into 3 groups according to ages: group $A$, age $50-70$ years $(n=50)$; group $B$, age 71-80 years $(n=50)$; group $C$, age $>80$ years $(n=850)$. After recording gender, body mass index, results of lung function testing, preoperative SBP, preoperative peripheral capillary oxygen saturation. Infusion of propofol was continued until the end of the procedure. 


\section{B. Medication and Monitoring}

Pharyngeal anesthesia was performed by using an $2 \%$ topical lidocaine spray before intravenous infusion of the sedative drugs. Propofol was administered intravenously by using the Diprifusor system which is a TCI system using the pharmacokinetic kinetic parameter set according to the Marsh model. The initial setting of the target blood concentration of propofol (1\% Diprivan Injection-kit; AstraZeneca) was set at $2.0 \mu \mathrm{g} / \mathrm{mL}$ for the nonelderly patients $<70$ years old)[7].The initial setting for the elderly patients ( $>70$ years old) was $1.0 \mu \mathrm{g} / \mathrm{mL}$, which was chosen for moderate sedation based on a previous study[8]. The blood concentration of propofol at each time point was calculated automatically and shown on the monitor of the TCI pump. For the objective measurement of the level of consciousness in sedated patients, the A2000 BIS monitor (Aspect Medical Systems, Newton, Mass) was used. The BIS score was kept between 50 and 80 . In the procedure period, if the BIS score went beyond the range of $50-80$, the target blood concentration was changed by 0.2 $\mu \mathrm{g} / \mathrm{mL}$ each time. An additional bolus of 1 to $2 \mathrm{~mL}$ of propofol was given if the patient's movements were frequent. When the BIS score was less than 50 or an adverse event (SBP $<80 \mathrm{mmHg}$ or $\mathrm{Spo} 2<90 \%$ ) occurred, the target blood concentration of propofol was reduced by $0.2 \mu \mathrm{g} / \mathrm{mL}$ with an immediate increase in the intravenous drip or oxygen dose. All patients received $1 \mathrm{mg} / \mathrm{kg}$ fentanyl as an analgesic agent just before insertion of the endoscope. All patients received supplemental oxygen $(2 \mathrm{~L} / \mathrm{min})$ by nasal cannula during sedation and were kept in the lateral decubitus position. If hypoxemia occurred during the sedation, we performed chin lift on the patient and increased the oxygen dose.

The patients' pulse rate, blood pressure, electrocardiogram, and $\mathrm{Spo} 2$ were monitored with a bedside monitor during the procedure. Blood pressure was recorded every 5 minutes. Spo2 and heart rate were recorded continuously. All adverse events including hypoxemia $\left(\mathrm{Spo}_{2}<90 \%\right)$ and hypotension $(\mathrm{SBP}<80 \mathrm{mmHg}$ ), and the total propofol dose were recorded during the ERCP.

\section{Statistical Analysis}

Continuous variables are presented as the median and range or interquartile range (IQR). Comparison of continuous variables was performed by the Mann-Whitney $U$ test, and comparison of dichotomous variables was made by using the Fisher exact test and logistic regression. To extract significant factors for each of major adverse events concerning propofol sedation( hypotension or hypoxemia), multivariate analyses were done by using logistic regression analysis. The significance level was set at $\mathrm{P}<0.05$. The resultant data were evaluated by using JMP software version 11 (SAS Institute, Cary, NC)

\section{RESULTS}

There were no statistically significant differences among the 3 groups in terms of American Society of Anesthesiologists classifications, pulmonary malfunction, underlying cardiovascular, pneurological disease and hypertension. There were no statistically significant differences between groups in gender, body mass index, procedure time (see Table 1) .

The older age groups needed a lower median amount of each target concentration. Median target concentration are respectively $2.6 \mu \mathrm{g} / \mathrm{mL}, 2.1 \mu \mathrm{g} / \mathrm{mL}$ and $1.6 \mu \mathrm{g} / \mathrm{mL}$ in the three groups. The requirement for the total infusion doses of propofol was lower the older groups.(group A:median $43 \mathrm{ml}$, IQR, 30-62; group B: median 35ml, IQR, 23-48; group C: median 30 ml, IQR, 18-40; $\mathrm{P}<0.0001$,) (see Table 1)

In addition, there were no statistically significant differences among groups in the percentages of Spo2. Hypotension (as defined by $\mathrm{SBP}<80 \mathrm{~mm} \mathrm{Hg}$ ) tended to occur more often in the younger groups, but the difference was not significant (group A: $35.6 \%$; group B: $28.5 \%$; group C: $23.3 \%, \mathrm{P}=0.072)$. Hypoxemia occurred significantly more often in the older group, but the prevalence was low (group A: $0 \%$; group $\mathrm{B}$ : $2.6 \%$; group $\mathrm{C}: 5.5 \%, \mathrm{P}=0.001$ ). All patients recovered from hypoxemia within 30 seconds. Only 4 patients needed vasopressor drugs to recover from hypotension, and no patient needed mask assisstant breath or intubation. In addition, all patients were stable under good sedation. (see TABLE I)

TABLE I. CHARACTERISTICS OF PROCEDURES AND ADVERSE EVENTS

\begin{tabular}{|c|c|c|c|c|}
\hline characteristics & Group A (50-70year) & Group B(71-80year) & Group C ( $>80$ year) & $P$ value \\
\hline $\operatorname{BMI}\left(\mathrm{kg} / \mathrm{m}^{2}\right)$ & $22.4(20.5-24.4)$ & $22.8(21.0-25.0)$ & $22.5(20.7-24.8)$ & 0.076 \\
\hline procedure time $(\mathrm{min})$ & $43.5(34.8-72.3)$ & $48.0(38.0-88.2)$ & $46.0(37.6-90.4)$ & 0.093 \\
\hline Minimum concentration of propofol $(\mu \mathrm{g} / \mathrm{mL})$ & $1.8(1.4-2.0)$ & $1.6(1.2-1.8)$ & $1.3(1.0-1.7)$ & $<0.003$ \\
\hline Maximum concentration of propofol $(\mu \mathrm{g} / \mathrm{mL})$ & $3.3(2.6-3.8)$ & $2.9(2.4-3.6)$ & $2.6(2.0-3.2)$ & $<0.001$ \\
\hline Average concentration of propofol $(\mu \mathrm{g} / \mathrm{mL})$ & $2.6(2.2-3.2)$ & $2.1(1.7-2.8)$ & $1.6(1.5-2.5)$ & $<0.001$ \\
\hline Total infusion dose of propofol $(\mathrm{mL})$ & $43(30-62)$ & $35(23-48)$ & $30(18-40)$ & $<0.001$ \\
\hline Hypotention incidence(SBP $<80 \mathrm{mmHg})(\%)$ & 35.6 & 28.5 & 23.3 & 0.072 \\
\hline Hypoxemia incidence(Spo2<90\%)(\%) & 0 & 2.6 & 5.5 & 0.01 \\
\hline
\end{tabular}

\section{DISCUSSION}

Although deep sedation in general endoscopic treatment has been reported, data are limited on the monitoring of propofol infusion in older patients[8,9]. This report evaluated the efficacy of the TCI system for propofol sedation during ERCP in different age period elder patients. In our study, there was an inverse correlation between age and target concentration of propofol. The older the age group was, the lower was the required total infusion dose and the lower was the required 
average maintenance dose of propofol with the TCI system of sedation. With regard to adverse events, the proportion of those with hypotension was highest in group A, which was the youngest elder group, whereas the rate of hypoxemia was significantly higher in the older groups (group B and C), although the number of patients with this adverse event was small. The possible cause of hypoxemia in group $\mathrm{C}$ was that those patients had more frequent episodes of hypoxemia during natural sleep than those in group A. Hypotension mostly occurred in the maintenance period, whereas hypoxemia occurred in both the maintenance and recovery periods. Preoperative low SBP was associated with hypotension, and abnormal pulmonary function was associated with hypoxemia in the older patient groups. Propofol sedation in the elderly patients during ERCP with the TCI system was as safe as in the younger patient group.

Propofol is a non barbiturate sedative hypnotic. It has a favorable pharmacokinetic profile as the lipid solubility confers a quick onset and short recovery time. It has also anti-emetic, anticonvulsant, antipruritic and amnesic effects. The usefulness of propofol sedation in endoscopy has been reported [10] . In addition, the safety of propofol sedation for endoscopy in elderly patients was also reported [11]. Although it is extremely effective and potent, propofol is limited by a relatively high incidence of dose-dependent hypotension and respiratory depression [12]. Once cardiorespiratory inhibition has occurred because of excessive infusion of propofol, it is necessary to provide cardiorespiratory support with a ventilator until propofol is metabolized because there are no antagonists available. To avoid excessive infusion of propofol, we used the BIS monitoring system, which makes objective evaluation of the depth of sedation possible. BIS evaluates the association among the different parts of the electroencephalogram at various stages. Several studies of BIS monitoring of propofol sedation during endoscopic procedures showed its effectiveness for stable sedation[13].In the study, there was an inverse correlation between age and the target concentration of propofol to maintain BIS values below 80. As a result, it was possible to maintain stable sedation with a lower amount of propofol for elderly patients compared with younger patients by TCI system. It was shown that elderly patients require a lower amount of propofol to reach similar levels of sedation than younger patients undergoing complex upper endoscopic procedures [14] .This result suggests that strict control of infusion by the TCI computer-assited pump could decrease the dose of propofol in elderly patients.

In this study, hypotension events occurred most frequently in the maintenance period. In particular, low preoperative SBP was associated with hypotension independent of the age group. A previous study showed that propofol has weaker respiratory suppression and stronger circulatory suppression, with a $36 \%$ incidence of hypotension compared with $14 \%$ of midazolam [15]. Because of the short-acting characteristics of propofol, almost all patients recovered immediately with decreases in the dose of propofol without using a vasopressor drug. Contrary to our expectation, hypotension occurred at a higher rate in the younger patient group. It was previously shown that propofol-induced hypotension was more prominent in elderly patients when the propofol dose was identical in elderly and younger patients. The incidence of hypotension in younger patients might be explained by the more rapid rate of increase in propofol concentrations [16].We believe that our finding was because of the stability of the sedation state in the older patient groups through the TCI system and the higher preoperative SBP in the aged groups.

Our study has some limitations. Firstly, the initial target concentrations of propofol differed among groups A, B and C. The difference in the initial setting. might have resulted in an increased propofol dose in group A, causing hypotension in patients in that group. Furthermore, the synergic action of opioid drugs might have resulted in the occurrence of adverse events. We used fentanyl in our study,therefore, the synergic action of fentanyl might explain the occurrence of this adverse event, although, to our knowledge, there are no previous data on this issue.

In conclusion, our study revealed that propofol sedation with a TCI system is very effective in performing ERCP safely in elderly patients with a lower dose of propofol.However, there is not yet a standard method of propofol sedation in endoscopic treatment, especially in elderly persons. Further studies on a larger scale with a prospective, controlled design are needed to standardize sedation with propofol.

\section{REFERENCES}

[1] L Fanti, P Testoni. Sedation and analgesia in gastrointestinal endoscopy:what is new? World J Gastroenterol. 16(2010):2451-2457.

[2] L Fisher, R Jain, SO Ikenberry, et al. Complications of ERCP. Gastrointestinal endoscopy. 75( 2012):467-473

[3] K Khan, O Steven. Bo, Shen Stewarl L, et al. Sedation and anesthesia in GI endoscopy. Gastrointestinal endoscopy.68(2008):815-826.

[4] A Beeton, ERCP: sedation or anaesthesia?South Afr J Anaesth Analg.17(2011):124-125.

[5] RM Tackley, GT lewis, C Prys-Robert, et al.Computer controlled infusion of propofol .Br J Anaesth.62(1989):46-53.

[6] JE Mandel, E Sarraf.The variability of response to propofol is reduced when a clinical observation is incorporated in the control: a simulation study.Anesth Analg.114(2012):1221-1229.

[7] L Fanti, M Agostoni, PG Arcidiacono, et al. Target-controlled infusion during monitored anesthesia care in patients undergoing EUS: propofol alone versus midazolam plus propofol. A prospective double-blind randomized controlled trial. Dig Liver Dis .39(2007):81-86.

[8] A Imagawa, H Hata, M Nakatsu, et al. A target-controlled infusion system with bispectral index monitoring of propofol sedation during endoscopic submucosal dissection. Endosc Int Open.3(2015):E2-6.

[9] $\mathrm{T}$ Gotoda, C Kusano, M Nonaka, et al. Non-anesthesiologist administrated propofol (NAAP) during endoscopic submucosal dissection for elderly patients with early gastric cancer. Gastric Cancer .17(2014) : 686-691.

[10] A Horiuchi, Y Nakayama, M Kajiyama,et al. Safety and effectiveness of propofol sedation during and after outpatient colonoscopy. World J Gastroenterol .18(2012):3420-3425.

[11] A Kerker, C Hardt, HE Schlief, et al. Combined sedation with midazolam/propofol for gastrointestinal endoscopy in elderly patients. BMC Gastroenterol .10(2010):11.

[12] J Fechner, H Ihmen, D Hatterscherd, et al. Comparative pharmacokinetics and pharmacodynamic of the new propofol prodrug GPI 15715 and propofol emulsion.Anesthesiology.101(2004):626-639.

[13] SC Chen, DK Rex. An initial investigation of bispectral monitoring as an adjunct to nurse-administered propofol sedation for colonoscopy. Am J Gastroenterol. 99(2004):1081-1086. 
[14] JF Martinez, JR Aparicio, L Company, et al. Safety of continuous propofol sedation for endoscopic procedures in elderly patients. Rev Esp Enferm Dig.103(2011):76-82.

[15] T Yamagata, D Hirasawa, N Fujita, et al. Efficacy of propofol sedation for endoscopic submucosal dissection (ESD): assessment with prospective data collection. Intern Med .50(2011):1455-1460.

[16] GE Van Poucke, LJ Bravo, SL Shafer. Target controlled infusions: targeting the effect site while limiting peak plasma concentration. IEEE Trans Biomed Eng .51(2004):1869-1875. 